\title{
Searching for Psychological Predictors of Suicidal Ideation in University Students
}

\author{
Adelino António Gonçalves Pereira ${ }^{1}$ \\ Centro de Psicologia da Força Aérea \\ Francisco Manuel dos Santos Cardoso \\ Universidade de Trás-os-Montes e Alto Douro
}

\begin{abstract}
The present study aims to identify psychological predictors of suicidal ideation in university students. We collected a sample of 366 participants, representing a population of 7102 students from a university in northern Portugal $(95 \% \mathrm{CI})$. Both in the whole sample and in the intra-gender analysis, students with suicidal ideation revealed higher levels of depressive symptoms, loneliness, social anxiety and fears of abandonment, and lower levels of comfort with intimacy and trust in others. Loneliness and depression are significant predictors of suicidal ideation, with an odds ratio of 1.095 and 1.108, respectively. The results were consistent with those found in the literature, and call for more research and implementation of intervention protocols in university populations.
\end{abstract}

Keywords: psychological predictors, suicidal ideation, university students

\section{Investigando Preditores Psicológicos de Ideação Suicida em Estudantes Universitários}

\begin{abstract}
RESUMO - Este trabalho buscou investigar preditores psicológicos de ideação suicida em estudantes universitários. Coletaramse dados de 366 participantes, que representavam uma população de 7102 estudantes de uma universidade no Norte de Portugal $(95 \%$ CI). Tanto na amostra total como nas análises intra-gêneros, estudantes com ideação suicida revelaram maiores níveis de sintomas depressivos, solidão, ansiedade social e medo do abandono, bem como menores níveis de conforto com a intimidade e confiança nos outros. Solidão e depressão mostraram-se preditores significativos de ideação suicida, com uma odds ratio de 1,095 e 1,108, respectivamente. Os resultados foram consistentes com os encontrados na literatura e destacam a necessidade de pesquisas e da implementação de protocolos de intervenção junto à população universitária.
\end{abstract}

Palavras-chave: preditores psicológicos, ideação suicida, universitários

According to the World Health Organization (WHO, 2012), suicidal behavior is an international public health problem. Worldwide, the suicide rate is 11.4 deaths per 100,000 inhabitants (WHO, 2014), representing the third leading cause of death in individuals between 15 and 44 years, and the second cause of death in individuals between 15 and 19 (WHO, 2012). It has been estimated that by 2020, the number of suicides will increase to over a million and a half, representing one death every 20 seconds, and a suicide attempt every one or two seconds (Bertolote \& Fleischmann, 2002; Nock et al., 2008). Suicide is also responsible for more than 20 million disability-adjusted life years and is a considerable economic burden (WHO, 2003). As an example, in the United States of America (USA) the combined costs related to both medical care and loss of productivity represented a total value exceeding 44 billion dollars (Center for Disease Control and Prevention, 2010). Focusing on university populations alone, suicide is the second leading cause of death (Schwartz, 2006). Moreover, during university years, $12 \%$ of students reveal suicidal thoughts, with a cyclical manifestation in $2.6 \%$ of those students (Wilcox et al., 2010). In Portugal, 10.7\% of university students admitted thinking about suicide in the last year (Pereira \& Cardoso, 2015).

1 Address for correspondence: Centro de Psicologia da Força Aérea, Azinhaga dos Ulmeiros, Lisboa, Portugal. CEP: 1.649-020. E-mail: adelinogpereira@gmail.com
Suicidal ideation is associated with several psychological disorders, including depression (Nock et al, 2009). In this respect, Eisenberg, Gollust, Golberstein, and Hefner (2007) found that about $67 \%$ of university students who reported suicidal ideation also had a depressive disorder, the most common one being a major depressive disorder (about $43 \%$ ). Also, suicidal ideation tends to be more intense as the severity of the depression increases (Arria et al., 2009; Garlow et al., 2008), putting individuals at high risk of attempting and committing suicide (Gollust, Eisenberg, \& Golberstein, 2008). Rates of depression have been increasing in college and it is a significant cause of the students' subpar performance (American College Health Association, 2009) and college dropout (Gollust et al., 2008).

Another relevant topic of discussion in university students is social anxiety. Some studies reveal that about $20 \%$ of students screen positive for this disorder (Shah \& Kataria, 2010). It is a significant cause of impaired performance at university, making it difficult to face practical evaluations and pose questions in the classroom. At the same time, it promotes feelings of discomfort, which leads the person to avoid group debates, seminars or even presentations (Shah \& Kataria, 2010). Anxiety disorders are commonly related to suicidal behaviors (Nepon, Belik, Bolton, \& Sareen, 2010; Thibodeau, Welch, Sareen, \& Asmundson, 2013), as revealed by Sareen et al. (2005), who found that about $25 \%$ of individuals with suicidal ideation and $28 \%$ of those attempting suicide suffer 
from one of such disorders. These authors also found that every disorder in analysis, including social anxiety disorder, was significantly correlated to suicidal ideation and suicide attempt, at a cross-sectional and longitudinal level.

Additionally, it is well known that poor interpersonal relationships, isolation and social uprooting play an important role in life dissatisfaction, increasing the risk for suicidal ideation and suicide (Joiner, 2005). In this vein, some studies analyze the impact of loneliness in young people. For example, in university populations, loneliness was associated to interpersonal relationship satisfaction and life fulfillment (Bugay, 2007). Additionally, multinational studies reveal that loneliness significantly correlates to attempted suicide and mediates the relationship between suicide attempt and hopelessness, both in male and female teenagers (Page et al., 2006). Rudatsikira, Muula, Siziya and Twa-Twa (2007) also concluded that higher levels of loneliness are related to an increase in suicidal ideation. On the other hand, some studies revealed that loneliness does not predict suicidal ideation (Lasgaard, Goossens, \& Elklit, 2010), suggesting the need to deepen the knowledge regarding these variables. The association between suicidal ideation and loneliness is not yet fully understood (Lasgaard et al., 2010) and few studies focus on the research about loneliness during adolescence (Page et al., 2006).

A relevant component of loneliness is related to the attachment to significant others (Dykstra \& Fokkema, 2007). Accordingly, attachment styles are central to interpersonal relationships and can perform a fundamental role in university. The quality of peer relationships is associated with academic, social, personal-emotional adjustment and institutional attachment (Buote et al., 2007). Peter, Roberts, and Buzdugan (2008) mention that depressive or anxiety symptoms are correlated to negative attachment to parents. In relation to suicidal behavior, negative attachment to parents is associated with higher levels of suicidal ideation (Davaji, Valizadeh, \& Nikamal, 2010; Peter et al., 2008), whereas a positive attachment to family members relates to lower levels of suicidal thoughts and significantly reduces suicide attempts among teenagers (Davaji et al., 2010; Maimon, Browning \& Brooks-Gunn, 2010). Lessard and Moretti (1998) added some interesting data to this topic, finding that suicidal ideation maintains a positive correlation with attachment styles with a negative model of self (preoccupied and fearful), deriving the opposite correlation when it comes to styles which maintain an inward positive model (secure and dismissing).

Taking into account these findings, the present work intends to contribute to the clarification of the psychological predictors of suicidal ideation, allowing for the development and improvement of effective and efficient warning systems capable of deriving timely interventions and minimizing acts of attempted or completed suicide. Thus, this study has two main objectives: (a) to compare students with and without suicidal ideation in terms of the overall levels of depressive symptoms, loneliness, social anxiety and three domains of adult attachment in interpersonal relationships: confidence in others, comfort with intimacy and fears of abandonment; and (b) to identify the best predictors of suicidal ideation, among the aforementioned variables.

\section{Method}

\section{Participants}

We collected a sample of $366($ female $=233 ;$ male $=133)$ university students from a population of $7,102(95 \% \mathrm{CI})$. The average age was $20.86(S D \pm 3.75)$ years for the females and $21.65(S D \pm 4.45)$ years for the males. At the time of data collection, $66.4 \%$ of students lived with classmates, $23.8 \%$ lived with their families or guardians, $8.5 \%$ lived alone, and $1.4 \%$ lived with their landlords.

\section{Instruments}

The Suicidal Ideation Questionnaire (SIQ). This questionnaire was developed by Reynolds, in 1987, and is composed of 30 items with differing severity levels assessing suicidal thoughts. Individuals must provide an answer using a seven-point Likert type scale. The sum of the items results in a score ranging from 0 to 180 , with higher scores meaning a higher frequency of suicidal thoughts. However, the original author stated that a score equal to or greater than 41 points represents a significant risk of suicidal acts and of suffering from psychopathology (Mazza \& Reynolds, 2001). The Portuguese version of the instrument was developed by Ferreira and Castela (1999), showing good psychometric properties. In this version, the item-total correlation values vary between .21 and .86 , however most correlations fall between .70 and .80 . Concerning convergent and divergent validity, the SIQ presented a positive and significant correlation with the Beck Depression Inventory, and a negative and significant correlation with the Rosenberg SelfEsteem Scale. Furthermore, the authors obtained a factormatrix with three distinct factors, which was reasonably akin to the results obtained by the author of the original scale. The present study revealed a Cronbach's alpha of .96 and item-total correlations ranged between .35 and .83 with an average of .65 .

Inventory of Depressive Symptomatology (IDS). This inventory was developed by Rush, Giles, Schlesser, Fulton, Weissenburger and Burns in 1986, in self-report and clinician-rated versions. Both are composed of 30 items, with four optional answers each, evaluating all of the domains included in the Diagnostic and Statistical Manual, Fourth Edition (DSM-IV) diagnosis of a Major Depressive Episode. For the present study, the self-rated version was adopted; for scoring and interpretation issues, items 11 and 12 were grouped into one item, labeled appetite changes, and items 13 and 14 were grouped into another item, called weight changes, composed of 28 items in total. The resulting scores can be qualitatively organized into five categories that indicate either the absence of depressive symptoms, or their presence in several differing levels of severity: scores of 0 - 13 correspond to the absence of depressive symptoms, 14 - 25 correspond to mild symptoms, 26 - 38 correspond to moderate symptoms, 39 - 48 correspond to severe symptoms, and $49-84$ correspond to very severe symptoms. The IDS research version was adapted to the Portuguese population 
by Pereira and Cardoso (2012), with a Cronbach's alpha of .87. The item-total correlations ranged between .13 and .64. Principal component analysis was performed, and the items whose item-total correlation was smaller than .30 were deleted. This resulted in a composite of two factors: 14 items related to affective and cognitive-emotional symptoms, and seven items related to sleep and somatic symptoms. Internal consistency for this version, determined using Cronbach's alpha, was .84; item-total correlations improved to between .22 and .63 , with an average of .44. It was considered, therefore, that this final version had acceptable psychometric properties for research purposes.

The University of California at Los Angeles' Loneliness Scale (UCLA Loneliness Scale). This scale was proposed by Russell, Peplau, and Ferguson (1978), based on the assumption that loneliness was a psychological state, and a unidimensional construct, as opposed to the idea that it may be a personality trait and a multidimensional phenomenon. The Portuguese adaptation of the scale was made by Neto (1989), using a sample of 286 students. In this adaptation, two items which composed the American version ended up being eliminated because they compromised the scale's psychometric proprieties. The registered Cronbach's alpha value in that study was .87. Furthermore, the scale correlated significantly with other constructs, such as anxiety, anguish, rejection and shame, among others. In the present study, the scale revealed good reliability, since it presents a Cronbach's alpha of .89 and item-total correlations that vary between .19 and .67 , with an average of .53 . We choose as reference the total score of the scale to evaluate loneliness.

Social Anxiety Questionnaire for Adults (SAQA). This questionnaire was developed by Caballo and CISO-A Research Team, and was validated through population samples of 18 differing Latin-origin countries, among them Portugal (Caballo et al., 2008; Caballo et al., 2010). It consisted of 30 direct items, to which one must respond using a 5-point Likert scale, and assign a total score that indicates the level of discomfort, tension or nervousness when faced with a set of social situations. The studies conducted so far suggest that this instrument presents excellent psychometric properties. The analysis of the factor structure has made evident the presence of five factors: Public Speaking/Interacting with Authority Figures; Interacting with Strangers; Interaction with the Opposite Sex; Assertive Expression of Displeasure; and Being Made Evident or "Playing the Fool". Furthermore, it presented a Cronbach's alpha of .91 (Caballo et al., 2010). In our own study, it revealed an internal consistency index of .95 and the item-total correlations varied between .46 and .73 , with an average of .61.

The Adult Attachment Scale - Revised (AAS - R). This scale was developed by Collins and Read in 1990, and consists of 18 items which participants must answer using a five-point scale, organized into three factors that assess different attachment characteristics: Anxiety/Fear of Abandonment, Trust in Others, and Comfort with Intimacy. This instrument demonstrated acceptable psychometric properties for each factor, and the importance of using these three sub-scales was justified by their clinical relevance (Canavarro, Dias, \& Lima, 2006; Collins \& Read, 1990; Hazan \& Shaver, 1987). Canavarro developed the Portuguese adaptation in 1997. Initially, the author obtained three factors that were designated as Anxious, Avoidant, and Secure. With further analysis, the dimensions ended up presenting another arrangement and, consequently, their current designation was adopted (Anxiety, Trust in Others, and Comfort with Intimacy) (Canavarro et al., 2006). In the study conducted by Canavarro et al. (2006), the item-total correlation, in general, proved acceptable. However, the results of the Cronbach's alpha were only satisfactory for the Anxiety factor (.84; and in the remainder .67 and .54). Also, the correlation between the three dimensions demonstrated that the Anxiety factor was negatively correlated with the factors of Comfort with Intimacy and Trust in Others. A positive correlation was verified between these last two.

In the actual study, the internal consistency analysis pointed to very similar results to those obtained by Canavarro et al. (2006), but were slightly more satisfactory. The factor, Anxiety, had a Cronbach's alpha of .86 and item-total correlations ranged between .51 and .75 , averaging .65 . The factor, Confidence in Others, had a Cronbach's alpha of .73, and the item-total correlations ranged between .40 and .57 , with an average of .48. Finally, the factor, Comfort with Intimacy, had a Cronbach's alpha of .59 and item-total correlations ranging between .20 and .40 , with an average of .33. Thus, in the present investigation, the total score for each factor was considered.

\section{Procedure}

Data collection. After obtaining approval and authorization from the academic authorities, the classes were informed about the purpose of the study and the ethical considerations related to confidentiality and their freedom to decide about participation, as well as discontinuing participation at any time. Students were also asked to check, after finishing the survey, whether all the items had been answered, in order to avoid missing values. We only collected data once in each course, randomly selecting a specific course year (from first year to the fifth) and assuring that all the years of study would be represented. In total, during a three months period, 21 different courses were included in the study.

No reward was given for participation in the study, despite of which only a residual percentage of students opted not to collaborate. Due to the nature of the investigation, they were also provided with the contacts of the researchers in case they felt the need for support, or they could head directly to the University's healthcare services.

\section{Data Analysis}

Comparison between individuals with and without suicidal ideation. The distinction between individuals with and without significant suicidal ideation was made using the cutoff recommended by the author of the SIQ, whereby students with a score equal to or less than 40 were considered to have non-significant suicidal ideation (NSI) and students with a score equal to or greater than 41 were considered to have significant suicidal ideation (SSI). We analyzed the 
distribution of the data in order to determine which statistics to use. T-test and Cohen's $d$ (Cohen, 1988) were used where the conditions for the adoption of parametric statistics were fulfilled. If not, the Mann-Whitney and Cliff's Delta $(\delta)$ were used. Romano, Kromrey, Coraggio, Skowronek, and Devine (2006) proposed that a Cliff's Delta equal to or larger than 0.147 is significant. Furthermore, the authors indicated that a value between 0.147 and 0.32 corresponded to a small effect size, a value between 0.33 and 0.473 corresponded to a medium effect size, and a value corresponding to, or exceeding, 0.474 represented a large effect size. The Cliff's Delta was calculated using the software, Cliff's Delta Calculator (CDC), developed by Macbeth, Razumiejczyk, and Ladesma (2011), and the Cohen's $d$, using the Excel file developed by Thalheimer and Cook (2002).

Predictive ability of psychological variables on suicidal ideation. For this analysis, logistic regression was applied, as the variables in analysis did not follow a normal distribution in the present study, justifying the use of nonparametric statistics. In this procedure, the $b$ coefficient of regression allows us to know the odds ratio value, which provides an indication of the change that occurs in the dependent variable as a consequence of the alteration of a unit in the independent variable. As such, the "logistic regression calculates changes in the log odds of the dependent, not changes in the dependent value as OLS regression does" (Burns \& Burns, 2008, p. 573). Wald's criterion will determine which variables are statistically significant predictors of suicidal ideation.

\section{Results}

\section{Comparison Between the SSI and the NSI Conditions}

The SSI condition exhibited significantly higher levels of depressive symptoms $(U=2141, p<.05, \delta=.692)$, loneliness $(U=2501, p<.05, \delta=.607)$, social anxiety $(t(364)=-4364, p<$ $.05, d=.74)$, and anxiety in affective relationships established with peers $(U=2792, p<.05, \delta=.562)$. In addition, the SSI condition revealed lower trust in others $(U=3,103, p<.05$, $\delta=-.513)$ and lower comfort with intimacy $(t(364)=3.532$, $p<.05, d=.62$ ).

\section{Comparison Between the SSI and the NSI Conditions of the Same Gender}

Males. As with the total sample, males with the SSI condition, compared with peers of the same gender with the NSI condition, scored significantly higher on levels of loneliness $(U=234, p<.05, \delta=.619)$, social anxiety $(U=306$, $p<.05, \delta=.502)$, depressive symptoms $(U=176.5, p<.05$, $\delta=.713)$, and anxiety in affective relationships $(U=285.5$, $p<.05, \delta=.535)$. On the other hand, they scored lower in trust in others $(U=385, p=.049, \delta=-.373)$ and comfort with intimacy $(U=458.5, p<.05, \delta=-.255)$. In the latter variable, the difference found was not statistically significant, however the effect size $(\delta=-.255$; small effect size) indicated that one should not neglect the existence of an effect produced by suicidal ideation.

Females. In the same way, females with the SSI condition revealed higher levels of loneliness $(U=1178.5, p<.05, \delta=$ $.602)$, social anxiety $(U=1827, p<.05, \delta=.382)$, depressive symptoms $(U=897, p<.05, \delta=.697)$ and anxiety in affective relationships $(U=1316.5, p<.05, \delta=.555)$. Concomitantly, they presented lower levels of trust in others $(U=1295.5, p$ $<.05, \delta=-.562)$ and comfort with intimacy $(U=1918, p<$ $.05, \delta=-.352)$ than females with the NSI condition.

\section{Predictive Analysis of Psychological Variables on Suicidal Ideation}

The predictive model is statistically significant, suggesting that the set of predictive variables is able to distinguish between the NSI and the SSI conditions (ChiSquare $=79.351, p=.000, d f=6$ ).

The Nagelkerke's $R^{2}$ reveals that the predictive variables explain $39.6 \%$ of the students' suicidal ideation. The model can correctly predict $90.2 \%$ of the data $(97.6 \%$ of the NSI and $28.2 \%$ of the SSI).

According to Wald's criterion, we verified that only the depressive symptomatology $(p=.000)$ and loneliness $(p=.011)$ are statistically significant predictors of suicidal ideation. The odds ratio is 1.108 for depressive symptomatology and 1.095 for loneliness. These results suggest that for each unit increase in depression or loneliness, it is about 1.1 times more likely for a student to have suicidal ideation (see Table 1).

Table 1. Regression Model for Psychological Predictors of Suicidal Ideation

\begin{tabular}{lcccccc}
\hline \multicolumn{1}{c}{ Predictor } & $\boldsymbol{B}$ & $\boldsymbol{S E}^{\boldsymbol{a}}$ & Wald & $\boldsymbol{d}^{\boldsymbol{b}}$ & $\mathbf{E x p ( B )}$ & $\mathbf{E X P ( B ) ~ 9 5 \% ~ C I ~}$ \\
\hline Depressive simptomathology (IDS) & 0.103 & 0.029 & $12.889^{* * *}$ & 1 & 1.108 & {$[1.048,1.173]$} \\
Trust in others (AAS - R) & -0.128 & 0.066 & 3.718 & 1 & 0.880 & {$[0.772,1.002]$} \\
Comfort with intimacy (AAS - R) & 0.112 & 0.081 & 1.917 & 1 & 1.119 & {$[0.954,1.311]$} \\
Anxiety (AAS - R) & 0.085 & 0.051 & 2.845 & 1 & 1.089 & {$[0.986,1.203]$} \\
Social anxiety (SAQA) & 0.003 & 0.014 & 0.054 & 1 & 1.003 & {$[0.976,1.031]$} \\
Loneliness (UCLA Loneliness Scale) & 0.091 & 0.036 & $6.423^{* *}$ & 1 & 1.095 & {$[1.021,1.175]$} \\
Constant & -8.680 & 3.204 & 7.340 & 1 & 0.000 & \\
\hline
\end{tabular}

Note. ${ }^{\mathrm{a}} S E-$ Standard Error; ${ }^{\mathrm{b}} d f-$ degrees of freedom; $* * p<.01 ; * * * p<.001$ 


\section{Discussion}

The present investigation's focal point was to characterize a set of variables likely to emerge as predictors of suicidal ideation, in order to contribute to the development of early warning systems, potentially able to monitor young people at risk of dying by suicide. The results were very clear in pointing out differences between NSI and SSI conditions. The SSI condition had (a) higher scores on all variables that could be considered psychopathological or were associated with psychological suffering and, on the other hand, (b) had lower scores on variables that were indicators of psychological adjustment and satisfaction in interpersonal relationships. Specifically, the first distinct feature refers to depressive symptoms, social anxiety, fears of abandonment in significant interpersonal relationships, and loneliness; the second distinct feature refers to the confidence in others and the degree of comfort with intimacy. This characterization is supported in the literature that mentions significant differences in various psychological and psychopathological domains between individuals with and without suicidal ideation (Borges \& Werlang 2006; Dervic et al., 2007; Eisenberg et al., 2007; Ellis \& Ratliff, 1986; Eskin, Voracek, Stieger, \& Altinyazar, 2011; Garlow et al., 2008; Gençöz \& Or, 2006; Kashden, Fremouw, Callahan, \& Franzen 1993; Lasgaard et al., 2010; Mazza \& Reynolds, 2001; Özer, Yildirim, \& Erkoç, 2015; Peter et al., 2008; Reinherz, Tanner, Berger, Beardslee, \& Fitzmaurice, 2006; Tyssen, Vaglum, Grønvold, \& Ekeberg, 2001).

The data showed very significant levels of psychological maladjustment in individuals with suicidal ideation, reflecting and impairing multiple domains of life. In the same line of concern, Reinherz et al. (2006) added interesting and relevant data from a longitudinal perspective. In a follow-up of over 25 years, they found that adolescents with suicidal ideation were, at the age of 30 , more than twice as likely to suffer from an Axis I disorder, about 12 times more likely to attempt suicide, and presented low self-esteem, significant interpersonal problems and impaired overall functioning. Simultaneously, these individuals seemed to experience more negative events throughout life (Horesh, Nachshoni, Wolmer, \& Toren, 2009; Liu \& Tein, 2005; Thompson et al., 2012).

In the present study, depressive symptoms seem to be the most distinctive differentiation between the SSI and the NSI conditions, followed by feelings of loneliness. Regarding the importance of loneliness, in the study by Page et al. (2006), the authors indicated that the relevance of this variable was associated with the collectivist feature of the participants' cultures. However, the data from this study indicate that even in a society with more individualistic traits, feelings of loneliness have a significant psychological impact.

The logistic regression confirmed the relationship that suicidal ideation maintains with depression and loneliness, since these variables are the only ones that have a statistically significant contribution for the regression model; an increase of one unit in the levels of loneliness or depression indicated that a subject had a higher chance of presenting significant suicidal ideation. In fact, these two variables are interrelated (Lasgaard et al., 2010) and, opposing what might eventually be considered, the relationship between these variables appears to have a significant impact on the well-being and mental health of both adolescents and young adults.

The results obtained within this study are in accordance with the general literature. Borges and Werlang (2006) verified that a person presenting depressive symptoms is nine times more likely to have suicidal ideation than one who does not present the same symptoms. The studies of Arria et al. (2009), and Wilcox et al. (2010), also indicated that depressive symptoms predict suicidal thoughts; Kirkcaldy, Eysenck, and Siefen (2004) verified the same capability of depressive symptoms and anxiety regarding suicidal ideation and self-harming behavior, both among men and women. One should further underline that in the study of Arria et al. (2009), even within a group with low-level depression, the predictive capability of those symptoms regarding suicidal ideation maintained their effect. Loneliness also showed its relevance as a risk factor for the manifestation of suicidal thoughts among non-university adolescents (Rudatsikira et al., 2007).

Given this data, it is relevant to discuss the intervention strategies in university students. Comprehensive and integrative approaches have already been developed specifically to this population. These approaches include all the domains of a student's life. Their intent is to prevent suicide, either by focusing on the university population or on students at risk, working with their families, peers, health professionals and university authorities. Based on the availability of mental health services, restriction of the means to die by suicide, development of personal skills and social networks, educational programs and crisis management facilities, these interventions are well developed in the USA. For detailed information, one may consult the work of the Suicide Prevention Resource Center (2004) and the National Mental Health Association \& The Jed Foundation (2002). However, in several countries, such as Portugal, there are no mental health programs for college students, which represent a relevant gap in this area. Some of these programs could be considered and adjusted for Portuguese university students, in order to fulfill a demand in healthcare related to suicidal behavior, as well as other mental health issues.

According to the findings of the present study, these programs should focus particularly on depression and loneliness, reinforcing prevention and treatment protocols. The existence of professional health care services in the university, with effective clinical treatment protocols, should be combined with a broader intervention and prevention strategy to make all the academic community aware of what depression is, its risk factors, consequences and how to identify and help students with depression. Also, universities should promote conditions for the positive integration of students into a new environment, in order to avoid social disconnection and isolation. The transition to college can lead to a significant decrease in contact with parents, peers and also with high school teachers, resulting in feelings of vulnerability and loneliness (Kraus-Parello, 2008). Overall, prevention of suicidal behavior in university students requires a holistic perspective, in which all the agents in the students' life are involved in the approach for prevention and treatment. 


\section{Conclusion}

The present study showed that individuals with suicidal ideation manifested psychological suffering more intensely. This happened for the entire sample, among males and females alike, in all dimensions evaluated. Specifically, students with suicidal ideation had higher levels of depressive symptoms, loneliness, social anxiety, and fears of abandonment. In addition, they had lower levels of comfort with intimacy and trust in others.

The predictive model pointed to the relevance of depressive symptoms and loneliness in predicting suicidal ideation. However, other variables not included in this study may have a significant impact on this condition. As mentioned by other authors, suicidal ideation in college students may have unique and varied etiologies. For that reason, it is difficult to create multidimensional models to explain this phenomenon.

The main limitation, or shortcoming, of this study was the fact that its data was collected in classrooms. It was possible that students who showed severe levels of psychopathology did not attend classes, and therefore were not even present for data collection. Finally, it is important to emphasize that there was a set of variables that were indeed likely to be monitored for purposes of prevention of suicidal behavior. These results present external validity since they are consistent with what was found in very different university settings, suggesting that this phenomenon manifests itself in very similar ways in different countries and that, therefore, the data are universal in nature and not characteristic of a particular country, region or university. However, more research studies are necessary in order to develop a better understanding of these phenomena as well as to design intervention strategies, meeting specific requirements of specific populations in order to better promote their psychological well-being.

\section{References}

American College Health Association. (2009). National College Health Assessment II: Reference Group Executive Summary - Fall 2008. Retrieved from http://www.acha-ncha.org/ docs/ACHA-NCHA_reference_group_executivesummary_ fall2008.pdf

Arria, A., O'Grady, K., Caldeira, K., Vincent, K., Wilcox, H., \& Wish, E. (2009). Suicide ideation among college students: A multivariate analysis. Archives of Suicide Research, 13(3), 230-246. doi:10.1080/13811110903044351

Bertolote, J., \& Fleischmann, A. (2002). Suicide and psychiatric diagnosis: A worldwide perspective. World Psychiatry, 1(3), 181-185.

Borges, V., \& Werlang, B. (2006). Estudo de ideação suicida em adolescentes de 15 a 19 anos. Estudos de Psicologia, 11(3), 345-351. doi:10.1590/S1413-294X2006000300012

Bugay, A. (2007). Loneliness and life satisfaction of turkish university students. Retrieved from www.ece.salford.ac.uk/ proceedings/papers/38_07.pdf
Buote, V., Pancer, S., Pratt, M., Adams, G., Birnie-Lefcovitch, S., Polivy, J., \& Wintre, M. (2007). The importance of friends: Friendship and adjustment among 1st-year university students. Journal of Adolescent Research, 22(6), 665-689. doi:10.1177/0743558407306344

Burns, R., \& Burns, R. (2008). Logistic regression. In R. Burns \& R. Burns (Eds.), Business research methods and statistics using SPSS (pp. 568-588). London: SAGE Publications Ltd.

Caballo, V., Salazar, I., Arias, B., Irurtia, M., Calderero, M., \& Equipo de Investigación CISO-A. (2010). Validación del "Cuestionario de Ansiedad Social para Adultos" (CASO-A30) en universitários españoles: Similitudes y diferencias entre carreras universitárias y comunidades autónomas. Behavioral Psychology/Psicología Conductual, 18(1), 5-34.

Caballo, V., Salazar, I., Irurtia, M., Arias, B., Hofmann, S., \& CISO-A Research Team. (2008). Social Anxiety in 18 nations: Sex and age differences. Behavioral Psychology/Psicología Conductual, 16(2), 163-187.

Canavarro, M., Dias, P., \& Lima, V. (2006). A avaliação da vinculação do adulto: Uma revisão crítica a propósito da aplicação da Adult Attachment Scale-R (AAS-R) na população portuguesa. Psicologia, 20(1), 155-186.

Center for Disease Control and Prevention. (2010). Cost of Injury Reports. Retrieved from Center for Disease Control and Prevention, Web-based Injury Statistics Query and Reporting System website: https://wisqars.cdc.gov:8443/costT/ ProcessPart1SetupServlet

Cohen, J. (1988). Statistical power analysis for the behavioral sciences (2nd ed.). Hillsdale: Lawrence Erlbaum Associates.

Collins, N., \& Read, S. (1990). Adult attachment, working models, and relationship quality in dating couples. Journal of Personality and Social Psychology, 58(4), 644-663. doi: 10.1037/0022- 3514.58.4.644

Davaji, R., Valizadeh, S., \& Nikamal, M. (2010). The relationship between attachment styles and suicide ideation: The study of Turkmen students, Iran. Procedia Social and Behavioral Sciences, 5, 1190-1194. doi: 10.1016/j.sbspro.2010.07.259

Dervic, K., Akkaya-Kalayci, T., Kapusta, N., Kaya, M., Merl, E., Vogel, E., ... Friedrich, M. (2007). Suicidal ideation among Viennese high school students. Wiener Klinische Wochenschrift, 119, 174-180. doi:10.1007/s00508-006-0753-4

Dykstra, P., \& Fokkema, T. (2007). Social and emotional loneliness among divorced and married men and women: comparing the deficit and cognitive perspectives. Basic and Applied Social Psychology, 29(1), 1-12. doi:10.1080/01973530701330843

Eisenberg, D., Gollust, S., Golberstein, E., \& Hefner, J. (2007). Prevalence and correlates of depression, anxiety, and suicidality among university students. American Journal of Orthopsychiatry, 77(4), 534-542. doi:10.1037/00029432.77.4.534

Ellis, T., \& Ratliff, K. (1986). Cognitive characteristics of suicidal and nonsuicidal psychiatric inpatients. Cognitive Therapy and Research, 10(6), 625-634. doi: 10.1007/BF01173750

Eskin, M., Voracek, M., Stieger, S., \& Altinyazar, V. (2011). A cross-cultural investigation of suicidal behavior and attitudes in Austrian and Turkish medical students. Social Psychiatry and Psychiatric Epidemiology, 46(9), 813-823. doi:10.1007/ s00127-010-0254-7 
Ferreira, J., \& Castela, M. (1999). Questionário de Ideação Suicida (Q.I.S.). In M. R. Simões, M. M. Gonçalves \& L. S. Almeida (Eds.), Testes e provas psicológicas em Portugal (vol. 2, pp. 123-130). Braga: APPORT/SHO.

Garlow, S., Rosenberg, J., Moore, D., Haas, A., Koestner, B., Hendin, H., \& Nemeroff, C. (2008). Depression, desperation, and suicidal ideation in college students: Results from the American Foundation for Suicide Prevention College Screening Project at Emory University. Depression and Anxiety, 25, 482-488. doi:10.1002/da.20321

Gençöz, T., \& Or, P. (2006). Associated factors of suicide among university students: Importance of family environment. Contemporary Family Therapy: An International Journal, 28(2), 261-268. doi:10.1007/s10591-006-9003-1

Gollust, S., Eisenberg, D., \& Golberstein, E. (2008). Prevalence and correlates of self-injury among university students. Journal of American College Health, 56(5), 491-498. doi: 10.3200/ JACH.56.5.491-498

Hazan, C., \& Shaver, P. (1987) Romantic love conceptualized as an attachment process. Journal of Personality and Social Psychology, 52(3), 511-524. doi: 10.1037/0022-3514.52.3.511

Horesh, N., Nachshoni, T., Wolmer, L., \& Toren, P. (2009). A comparison of life events in suicidal and nonsuicidal adolescents and young adults with major depression and Borderline Personality Disorder. Comprehensive Psychiatry, 50(6), 496-502. doi: 10.1016/j.comppsych.2009.01.006

Joiner, T. (2005). Why people die by suicide. Cambridge: Harvard University Press.

Kashden, J., Fremouw, W., Callahan, T., \& Franzen, M. (1993). Impulsivity in suicidal and nonsuicidal adolescents. Journal of Abnormal Child Psychology, 21(3), 339-353. doi: 10.1007/ BF00917538

Kirkcaldy, B., Eysenck, M., \& Siefen, G. (2004). Psychological and social predictors of suicidal ideation among young adolescents. School Psychology International, 25(3), 301-316. doi: $10.1177 / 0143034304046903$

Krause-Parello, C. (2008). Loneliness in the school setting. The Journal of School Nursing, 24(2), 66-70. doi: $10.1177 / 10598405080240020301$

Lasgaard, M., Goossens, L., \& Elklit, A. (2010). Loneliness, depressive symptomatology, and suicide ideation in adolescence: cross-sectional and longitudinal analyses. Journal of Abnormal Child Psychology, 39(1), 137-150. doi: 10.1007/ s10802-010-9442-x

Lessard, J., \& Moretti, M. (1998). Suicidal ideation in an adolescent clinical sample: Attachment patterns and clinical implications. Journal of Adolescence, 21(4), 383-395. doi: 10.1006/ jado.1998.0169

Liu, X., \& Tein, J.-Y. (2005). Life events, psychopathology, and suicidal behavior in Chinese adolescents. Journal of Affective Disorders, 86(2-3), 195-203. doi: 10.1016/j.jad.2005.01.016

Macbeth, G., Razumiejczyk, E., \& Ladesma, R. (2011). Cliff's Delta Calculator: A non-parametric effect size software for two groups of observations. Universitas Psychologica, 10(2), 545-555.

Maimon, D., Browning, C., \& Brooks-Gunn, J. (2010). Collective efficacy, family attachment, and urban adolescent suicide attempts. Journal of Health and Social Behavior, 51(3), 307324. doi: $10.1177 / 0022146510377878$
Mazza, J., \& Reynolds, W. (2001). An investigation of psychopathology in nonreferred suicidal and nonsuicidal adolescents. Suicide and Life-Threatening Behavior, 31(3), 282-302. doi: 10.1521/suli.31.3.282.24245

National Mental Health Association \& The Jed Foundation. (2002). Safeguarding your students against suicide: Expanding the safety net. Proceedings from panel on vulnerability, depressive symptoms, and suicidal behavior on college campuses. Retrieved from http://www.acha.org/topics/docs/ Safeguarding_Against_Suicide_FULLreport.pdf

Nepon, J., Belik, S., Bolton, J., \& Sareen, J. (2010). The relationship between anxiety disorders and suicide attempts: findings from the National Epidemiologic Survey on Alcohol and Related Conditions. Depression and Anxiety, 27(9), 791-798. doi: $10.1002 /$ da. 20674

Neto, F. (1989). Avaliação da solidão. Psicologia Clínica, 2, 65-79.

Nock, M., Borges, G., Bromet, E., Cha, C., Kessler, R., \& Lee, S. (2008). Suicide and suicidal behavior. Epidemiologic Reviews, 30, 133-154. doi: 10.1093/epirev/mxn002

Nock, M., Hwang, I., Sampson, N., Kessler, R., Angermeyer, M., Beautrais, A., ... Williams, D. (2009). Cross-National Analysis of the Associations among Mental Disorders and Suicidal Behavior: Findings from the WHO World Mental Health Surveys. PLoS Medicine, 6(8), 1-17. doi:10.1371/journal. pmed.1000123

Özer, Ü., Yildirim, E., \& Erkoç, S. (2015). Relationship of suicidal ideation and behavior to attachment style in patients with major depression. Archives of Neuropsychiatry, 52, 283-288. doi:10.5152/npa.2015.7459.

Page, R., Yanagishita, J., Suwanteerangkul, J., Zarco, E., Mei-Lee, C., \& Miao, N.-F. (2006). Hopelessness and loneliness among suicide attempters in school-based samples of Taiwanese, Philippine and Thai adolescents. School Psychology International, 27(5), 583-598. doi:10.1177/0143034306073415

Pereira, A., \& Cardoso, F. (2012). Adaptação portuguesa do Inventário de Sintomatologia Depressiva - Auto-resposta (Research Report). University of Trás-os-Montes and Alto Douro.

Pereira, A., \& Cardoso, F. (2015). Suicidal ideation in university students: Prevalence and association with school and gender. Paidéia (Ribeirão Preto), 25(62), 299-306. doi:10.1590/198243272562201503.

Peter, T., Roberts, L., \& Buzdugan, R. (2008). Suicidal ideation among Canadian youth: A multivariate analysis. Archives of Suicide Research, 12(3), 263-275. doi:10.1080/13811110802100882

Reinherz, H., Tanner, J., Berger, S., Beardslee, W., \& Fitzmaurice, G. (2006). Adolescent suicidal ideation as predictive of psychopathology, suicidal behavior, and compromised functioning at age 30. American Journal of Psychiatry, 163(7), 1226-1232. doi:10.1176/appi.ajp.163.7.1226

Romano, J., Kromrey, J., Corragio, J., Skowronek, J., \& Devine, L. (2006). Exploring methods for evaluating group differences on the NSSE and other surveys: Are the t-test and Cohen's $d$ indices the most appropriate choices? Paper presented at the Southern Association for Institutional Research, Arlington, Virginia.

Rudatsikira, E., Muula, A., Siziya, S., \& Twa-Twa, J. (2007). Suicidal ideation and associated factors among school-going adolescents in rural Uganda. BMC Psychiatry, 7(67), 1-6. doi: 10.1186/1471-244X-7-67 
Russell, D., Peplau, L., \& Ferguson, M. (1978). Developing a measure of loneliness. Journal of Personality Assessment, 42(3), 290-294. doi: 10.1207/s15327752jpa4203_11

Sareen, J., Cox, B., Afifi, T., Graaf, R., Asmundson, G., ten Have, M., \& Stein, M. (2005). Anxiety disorders and risk for suicidal ideation and suicide attempts: A population-based longitudinal study of adults. Archives of General Psychiatry, 62, 1249-1257. doi:10.1001/archpsyc.62.11.1249

Schwartz, A. (2006). College student suicide in the United States: 1990-1991 through 2003-2004. Journal of American College Health, 54(6), 341-352. doi: 10.3200/JACH.54.6.341-352

Shah, P., \& Kataria, L. (2010). Social phobia and its impact in Indian university students. The Internet Journal of Mental Health, 6(2). Retrieved from http://www.ispub.com/journal/the internet_journal_of_mental_health/volume_6_number_2_43/ article/social-phobia-and-its-impact-in-indian-universitystudents.html

Suicide Prevention Resource Center. (2004). Promoting mental health and preventing suicide in college and university settings. Retrieved from http://www.sprc.org/sites/sprc.org/files/library/ college_sp_whitepaper.pdf

Thalheimer, W., \& Cook, S. (2002). How to calculate effect sizes from published research: A simplified methodology. Retrieved from http://education.gsu.edu/coshima/EPRS8530/ Effect_Sizes_pdf4.pdf
Thibodeau, M., Welch, P., Sareen, J., \& Asmundson, G. (2013). Anxiety disorders are independently associated with suicide ideation and attempts: Propensity score matching in two epidemiological samples. Depression and Anxiety, 30(10), 947-954. doi:10.1002/da.22203

Thompson, R., Proctor, L., English, D., Dubowitz, H., Narasimhan, S., \& Everson, M. (2012). Suicidal ideation in adolescence: Examining the role of recent adverse experiences. Journal of Adolescence. doi:10.1016/j.adolescence.2011.03.003

Tyssen, R., Vaglum, P., Grønvold, N., \& Ekeberg, Ø. (2001). Suicidal ideation among medical students and young physicians: a nationwide and prospective study of prevalence and predictors. Journal of Affective Disorders, 64(1), 69-79. doi:10.1016/S0165-0327(00)00205-6

Wilcox, H., Arria, A., Caldeira, K., Vincent, K., Pinchevsky, G., \& O'Grady, K. (2010). Prevalence and predictors of persistent suicide ideation, plans, and attempts during college. Journal of Affective Disorders, 127(1-3), 287-294. doi:10.1016/j. jad.2010.04.017

World Health Organization. (2003). World Health Report. Shaping the Future. Retrieved from http://www.who.int/whr/2003/en/ whr03_en.pdf

World Health Organization. (2012). Public health action for the prevention of suicide. Geneva: World Health Organization.

World Health Organization. (2014). Preventing suicide: A global imperative. Geneva: World Health Organization.

Recebido em 09.10.2015

Primeira decisão editorial em 07.12.2016

Versão final em 23.01.2017

Aceito em 06.04.2017 